DOI: $10.2478 / \mathrm{v} 10122-010-0011-0$

\title{
GLOTTOCHRONOLOGICAL CLASSIFICATION OF OROMO DIALECTS
}

\author{
VÁCLAV BLAŽEK
}

\begin{abstract}
Aвstract. Václav Blažek. Glottochronological Classification of Oromo Dialects. Lingua Posnaniensis, vol. LII (2)/2010. The Poznań Society for the Advancement of the Arts and Sciences. PL ISSN 00794740, ISBN 978-83-7654-078-8, pp. 27-42

The purpose of the present article is the classification of the Oromo dialect continuum. Firstly, the previous attempts are discussed. As a method the so-called 'recalibrated glottochronology' developed by Sergei Starostin was applied. The results confirm the mutual relation of the 'mainstream' dialects, Maca, Qottu and Borana. In the case of more peripheral dialects the differences are bigger in comparison with the 'impressionistic' model sketched in Ethnologue ${ }_{16}$ (Bender compared only those three dialects). The reason probably consists in the chosen method: although it is not explicitly said in Ethnologue ${ }_{16}$, the classification used combines the qualitative and geographical approaches, contrary to the present model based on strictly quantitative principles.
\end{abstract}

Václav Blažek, Masaryk University, Brno, Czech Republic, blazek@phil.muni.cz

\section{INTRODUCTION}

The aim of the present study is to apply the so-called 'recalibrated glottochronology' to the Oromo dialect continuum. In number of speakers, Oromo represents surprisingly the second biggest Afroasiatic language with c. 30.000.000 speakers (Ethnologue, 16th ed.; according to census from 2007 there are 25.488.344 ethnic Oromos in Ethiopia - see Wikipedia). It is only one tenth of the people speaking the first Afroasiatic language in this order, Arabic. With regard to the relatively high number of speakers (abstracting from Arabic) and to the vast territory, where various Oromo dialects are spoken, it is natural to expect a relatively deep divergence between the geographically most distant dialects.

\section{PREVIOUS CLASSIFICATIONS OF OROMO DIALECTS}

2.1. In comparison with Somali (e.g. EHRET \& Ali 1984), the classification of the Oromo dialects is only in the beginning. In Ethnologue ${ }_{16}$, an outline of the Oromo dialect classification is only described. It may be transformed in the following tree-diagram: 


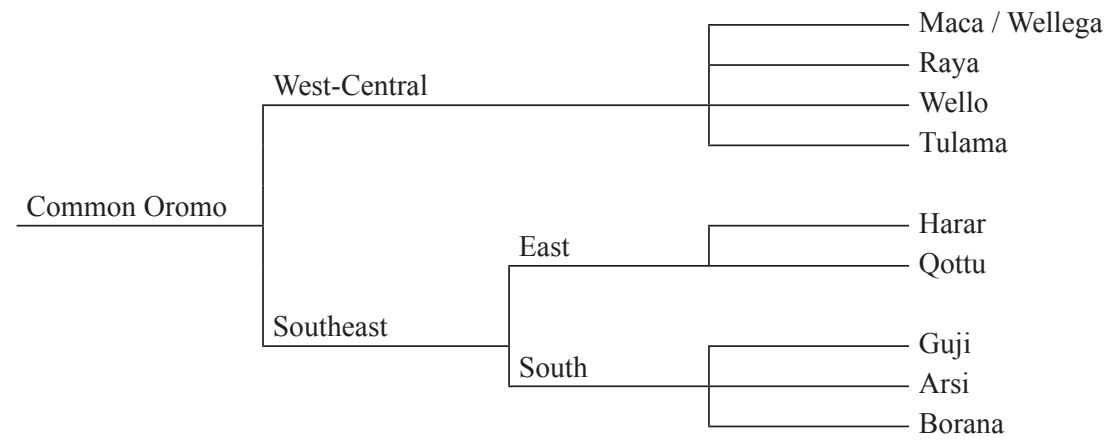

Scheme 1

2.2. The first attempt to apply the lexicostatistic method to measure the mutual distance of Oromo dialects, namely Maca, Qottu and Borana, was realized by BenDER (1971: 174; wordlists: 246-247). His results were Borana vs. Qottu 88\%, Maca vs. Qottu 81\%, Borana vs. Maca $79 \%$. It can be depicted in the following tree-diagram:

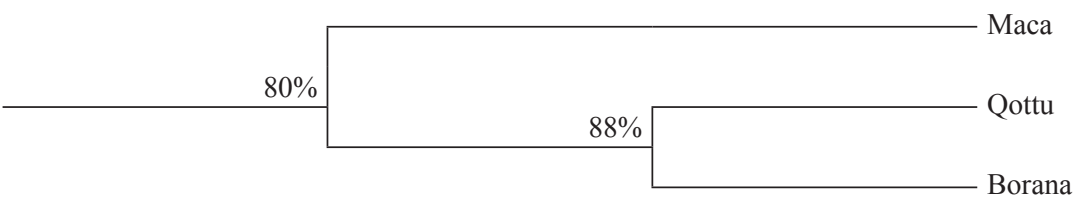

Scheme 2

\section{APPLICATION OF 'RECALIBRATED GLOTTOCHRONOLOGY' TO CLASSIFICATION OF THE OROMO DIALECTS}

3.1. In the present study the lexical data of 8 Oromo dialects recorded in the recent time of the last 4 decades were collected and arranged in agreement with etymological correspondences. Five of them appear in Scheme 1, Maca and Wellega are presented separately here. Orma and Waata are included in the Oromo classification for the first time. For Raya, Wello, Tulama and Arsi the relevant lexical material is not at our disposal.

3.2. The results of the mutual comparisons between Oromo dialects in the standard 100-word-list were summarized in Table 1.

Table 1

\begin{tabular}{|l|c|c|c|c|c|c|c|}
\hline & Harar & Maca & Guji & Qottu & Borana & Orma & Waata \\
\hline Wellega & $89 / 96=.927$ & $97 / 98=.990$ & $89 / 98=.908$ & $84 / 89=.944$ & $94 / 100=.94$ & $87 / 100=.87$ & $86 / 100=.86$ \\
\hline Harar & & $91 / 95=.958$ & $82 / 94=.872$ & $80 / 88=.909$ & $90 / 96=.938$ & $82 / 96=.854$ & $81 / 96=.844$ \\
\hline Maca & & & $89 / 98=.908$ & $83 / 88=.943$ & $93 / 98=.949$ & $90 / 98=.918$ & $88 / 98=.898$ \\
\hline Guji & & & & $81 / 88=.920$ & $91 / 97=.938$ & $86 / 97=.887$ & $84 / 97=.867$ \\
\hline Qottu & & & & & $85 / 89=.955$ & $80 / 89=.899$ & $76 / 89=.854$ \\
\hline Borana & & & & & & $94 / 100=.94$ & $92 / 100=.92$ \\
\hline Orma & & & & & & & $95 / 100=.95$ \\
\hline
\end{tabular}


3.3. These figures may be transformed in the following tree-diagram, including its glottochronological interpretation

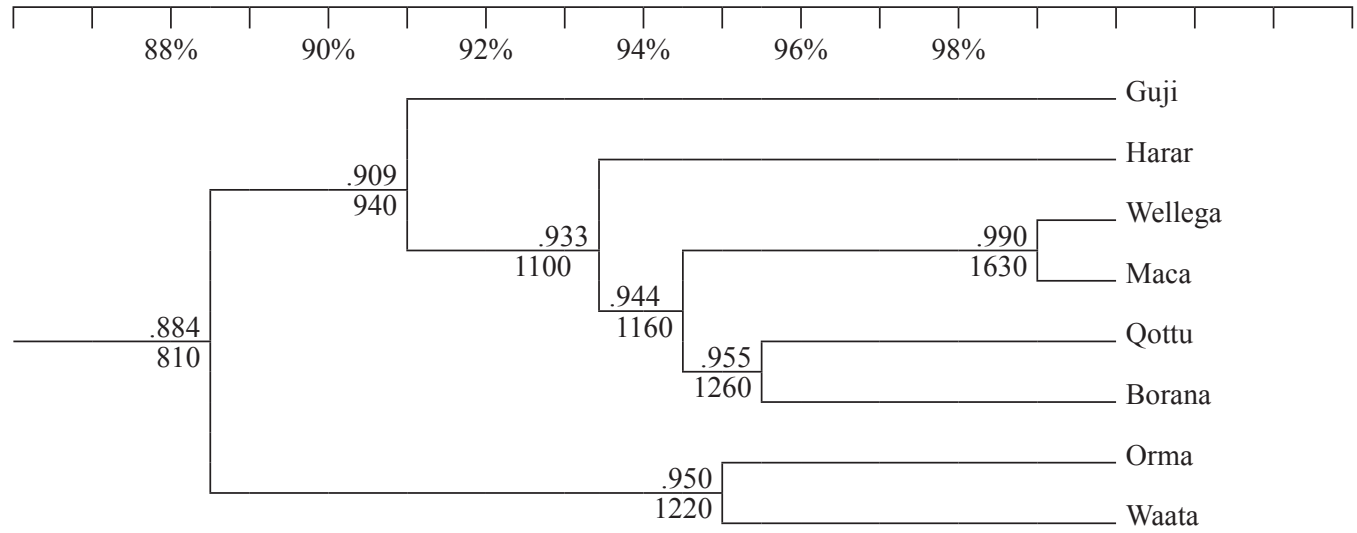

Scheme 3

\section{DISCUSSION OF THE PRESENT RESULTS}

The present results do not confirm the topology of the diagram in Scheme 1. There is only a partial agreement in the closer relation between Qottu and Borana in comparison of both with Maca / Wellega in Schemes 1 \& 2. The most important new information is the separated position of Orma and Waata, but not Borana which belongs to the mainstream of the Oromo dialects. The internal diversity in the Oromo dialect continuum is comparable with the Dullay cluster, if Tsamay is taken aside, or outside of Africa, with the Balto-Fennic languages.

\section{POSITION OF THE OROMO DIALECT CLUSTER WITHIN EAST CUSHITIC}

The East Cushitic branch of the Cushitic language family has been divided into the following groups: Afar-Saho, Somaloid, Galaboid, Oromoid, Dullay, Highland East Cushitic $=$ Burji-Sidamo, plus the isolated language Yaaku. Their internal relations, calculated according to the recalibrated glottochronology developed by Sergei Starostin (cf. BLAŽEK 1997, 2006; BLAŽEK \& ZА́ног̌́́ 2008[09]), are depicted in the tree-diagrams in the left side. In the right side above there is the tree-diagram depicting the arrangement of these groups in the higher hierarchy. This model modifies the idea of the unit called 'Omo-Tana', formulated by Bernd HeInE (1976: 179), who defined three subbranches: Western = Galaboid, Northern $=$ Bayso, Eastern $=$ 'Sam' $=$ Somali + Boni + Rendille + Jiddu. For comparison, the tree-diagrams expressing the internal relations of the Central Cushitic = Agaw and South Cushitic (minus Ma'a and Dahalo) are added.

The figures indicate the estimation of the year of disintegration $(-=\mathrm{BC}) /$ percentage of the common cognates from the standard 100-word-list. 


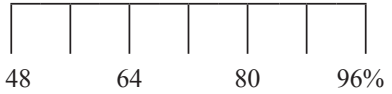

$1000 / \longdiv { 9 1 . 6 }$ Afar
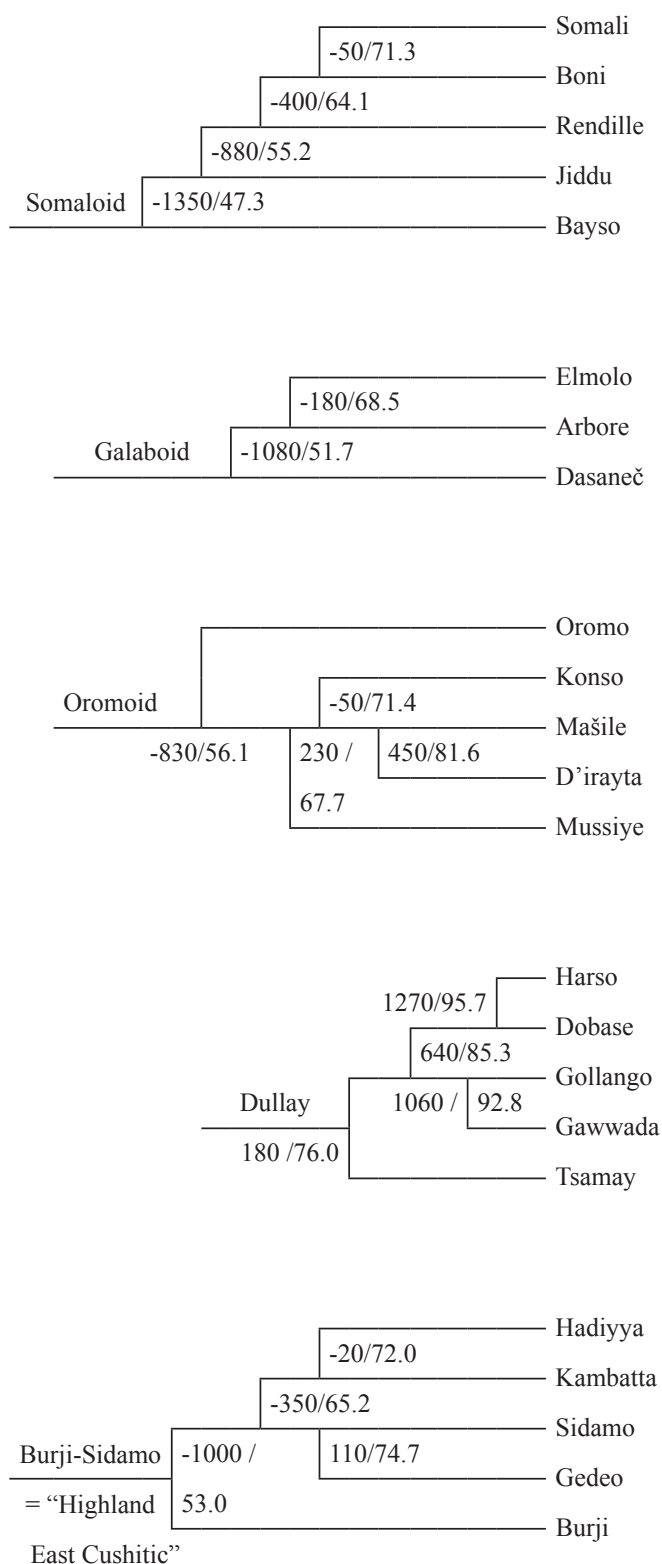

Dasaneč
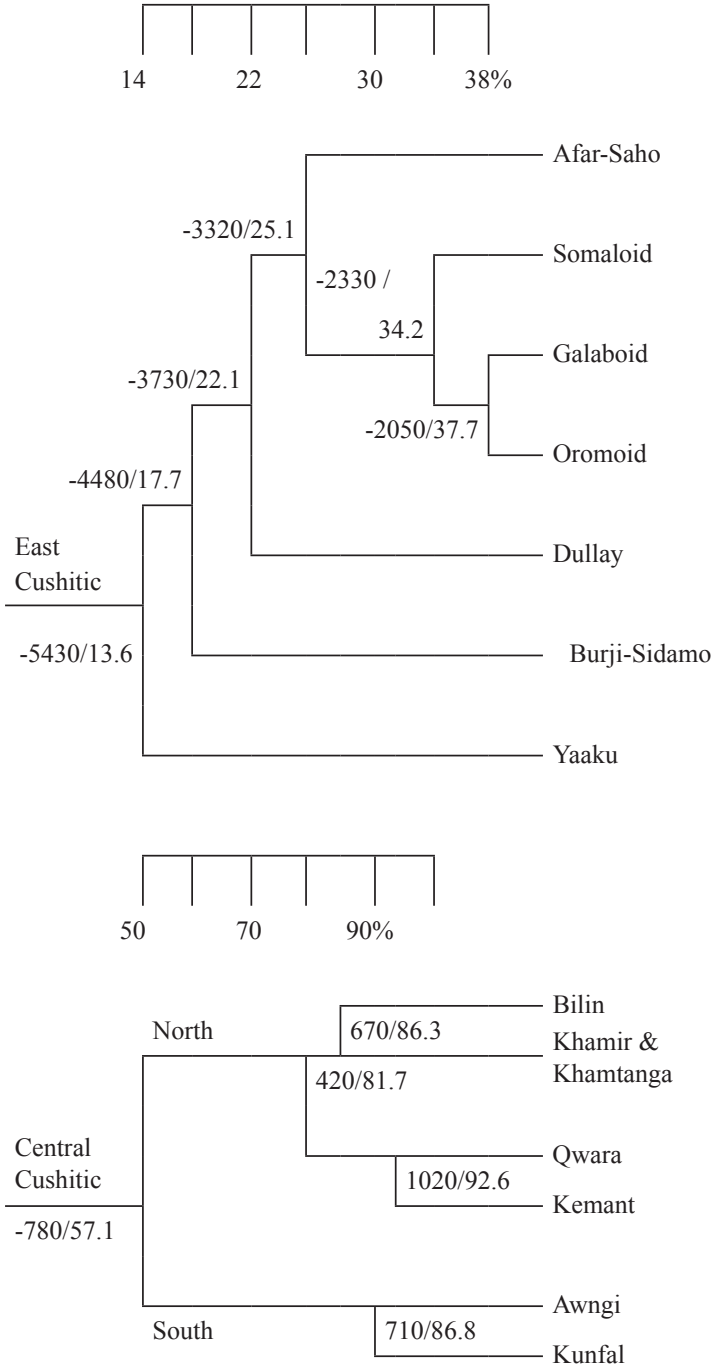

(without measure)

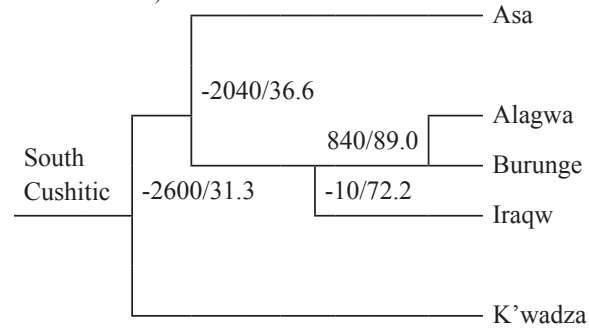




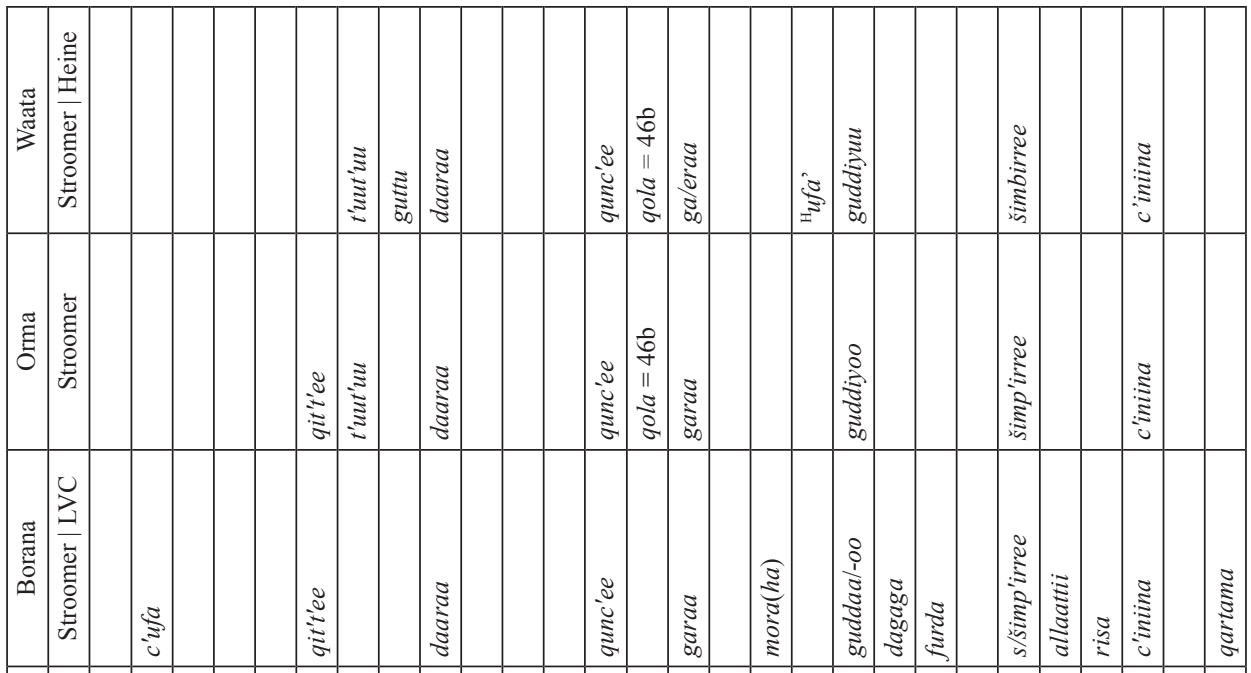

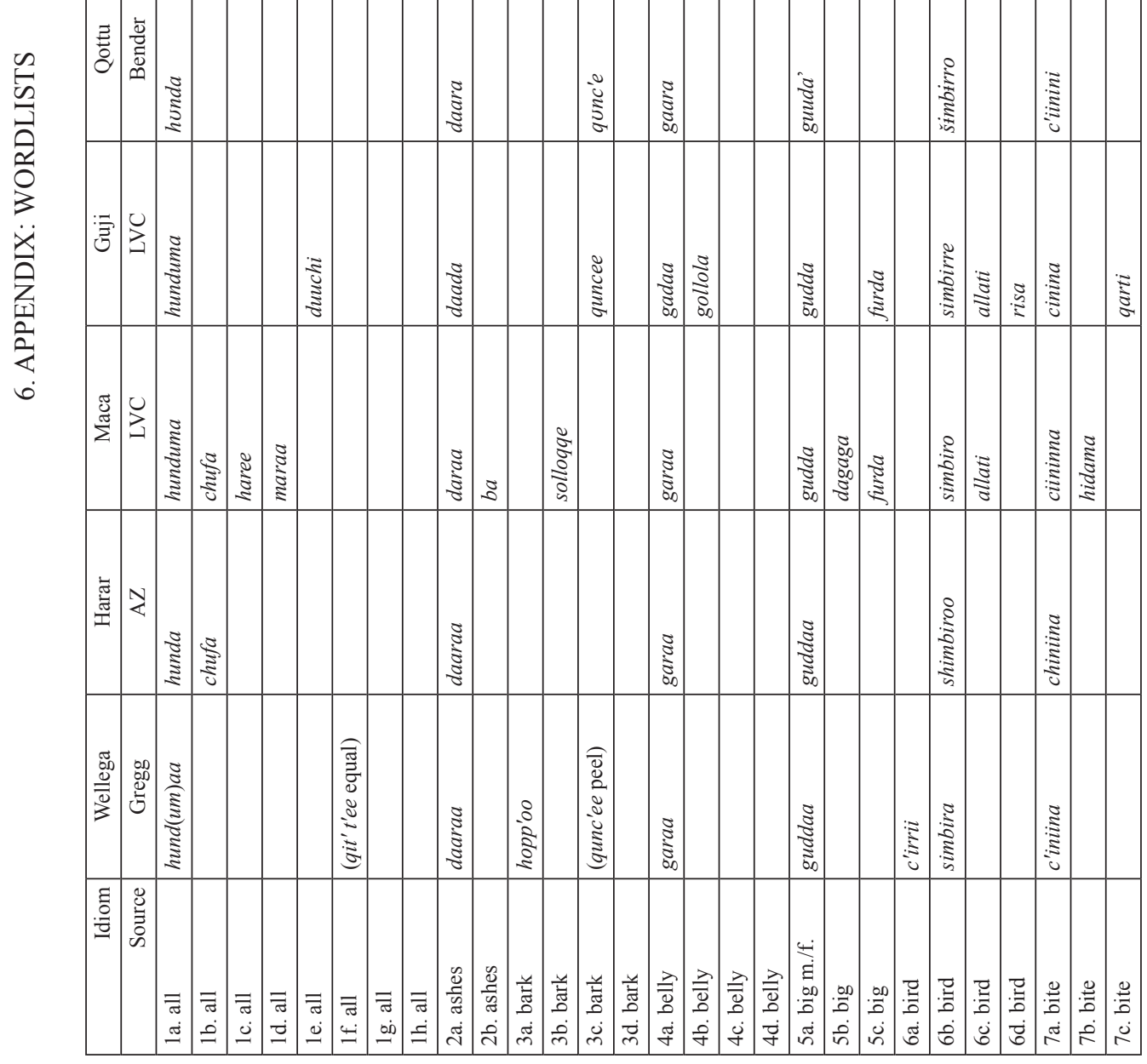




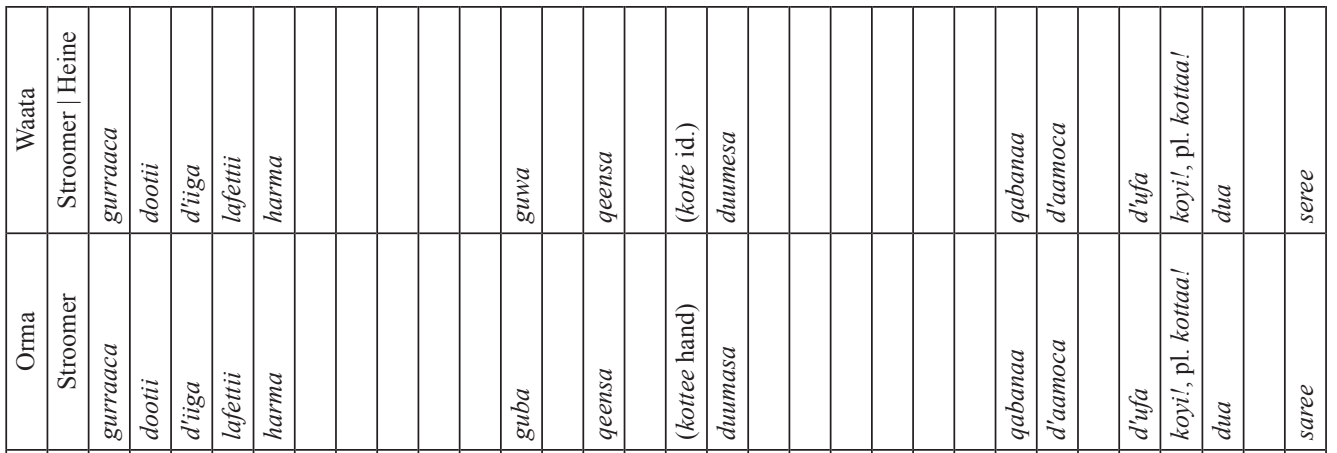

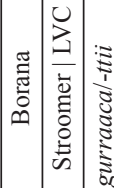

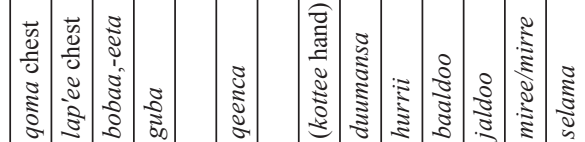

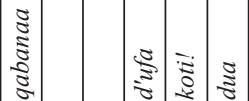

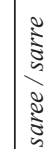

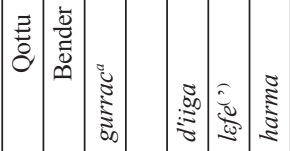

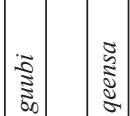

范

离

$\frac{\sqrt{3}}{2} \sqrt[3]{2}$

కั

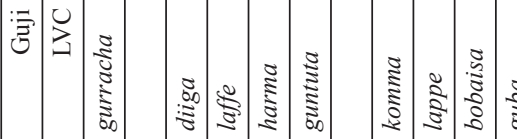

$\sqrt{\frac{5}{2}}$

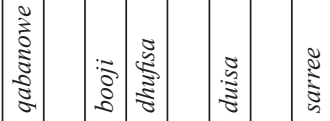

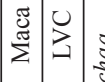

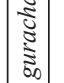

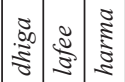

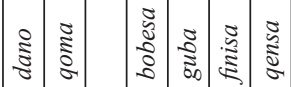

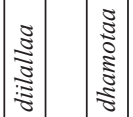

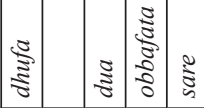

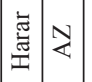

स 4

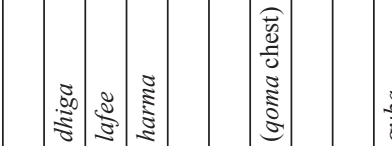

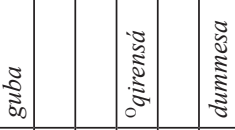

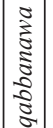

苛

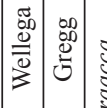

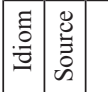

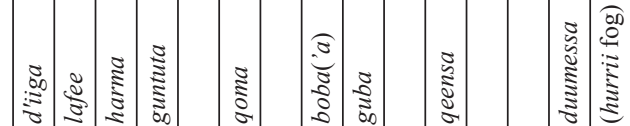

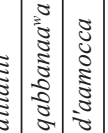

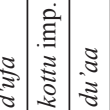

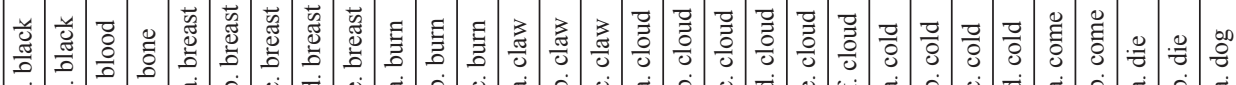

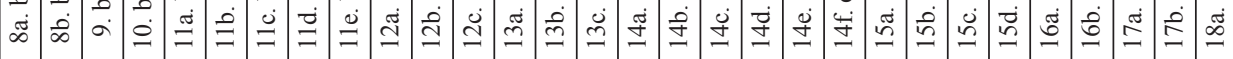




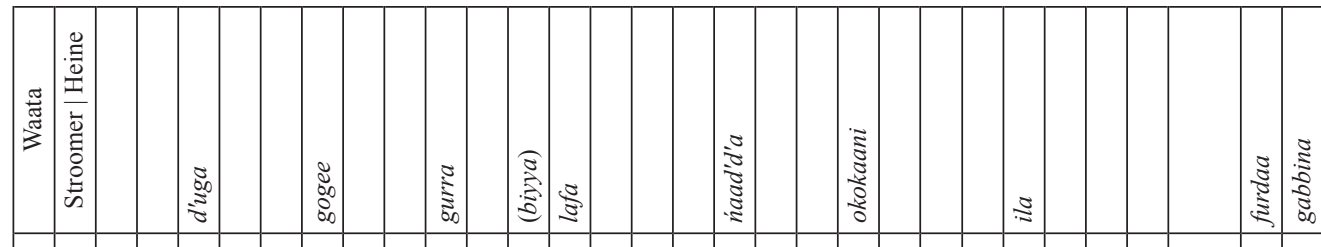

泀

$\stackrel{8}{\stackrel{8}{8}}$

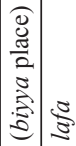

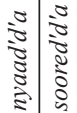

$\frac{\frac{9}{2}}{\frac{3}{3}}$

$\frac{\sqrt{5}}{3}$

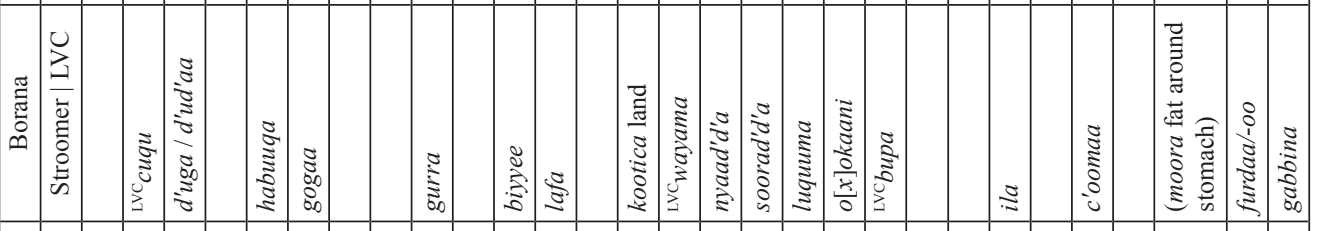

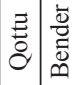

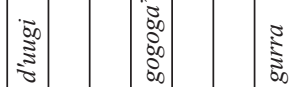

望

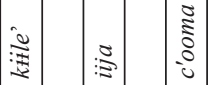

:

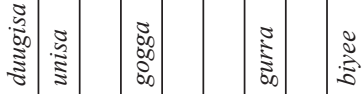

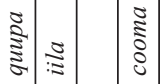

疍

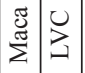

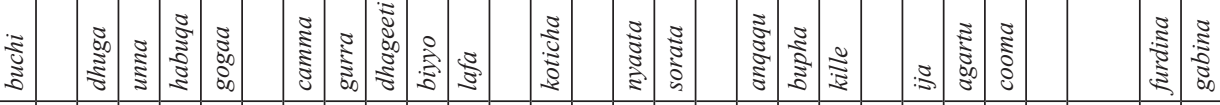

昰는

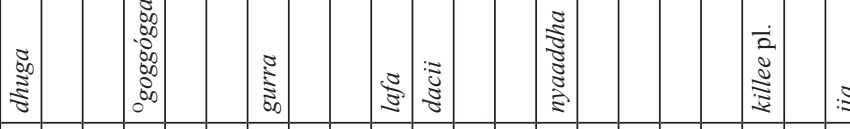

$\sqrt{2}$

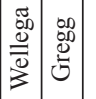

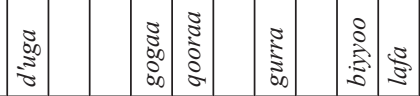

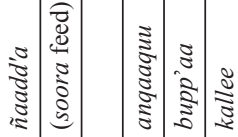

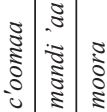

胥

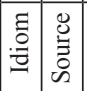

足

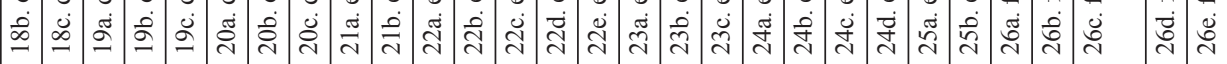




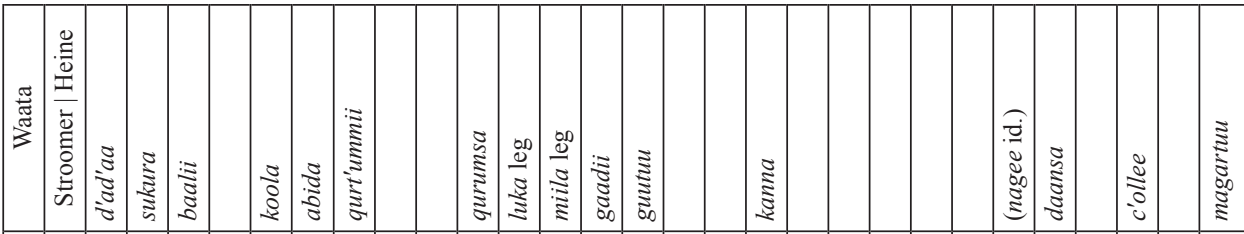

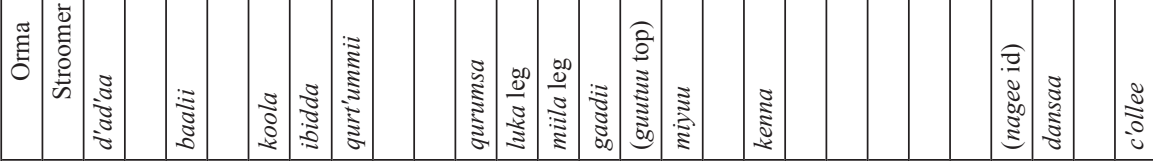

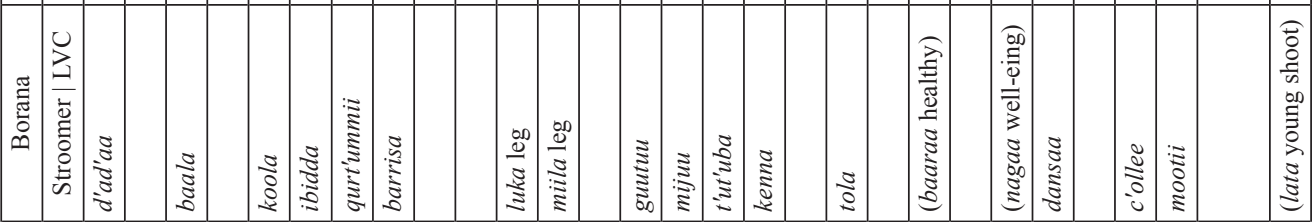

棓

$\frac{\sqrt{3}}{3}$

$\cong$

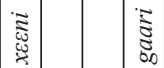

:

童产

言

童造

番彭

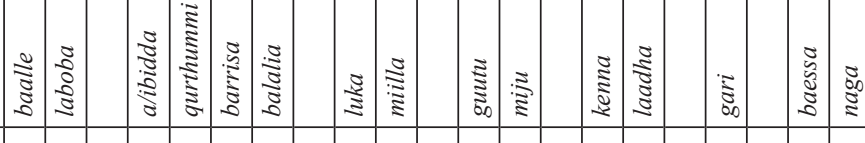

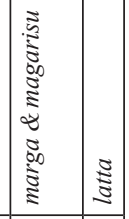

憲车

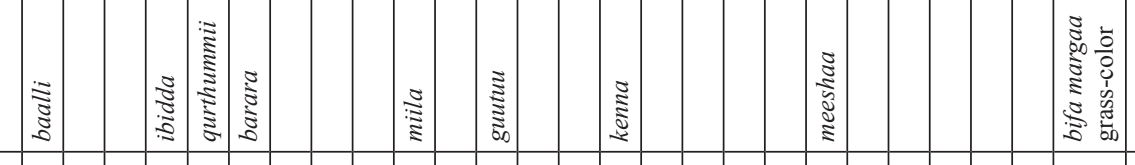

In

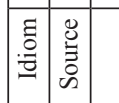

蛋童言

密

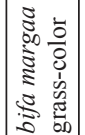

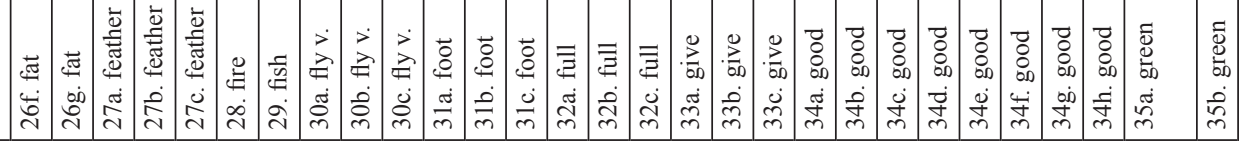




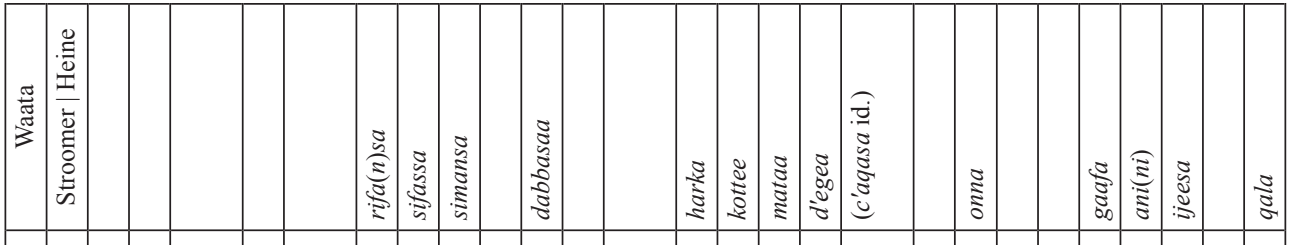

节言

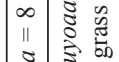

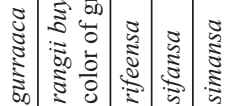

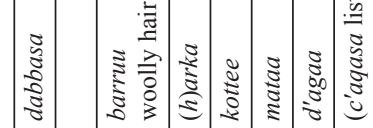

錘

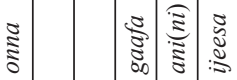

$\frac{2}{2}$

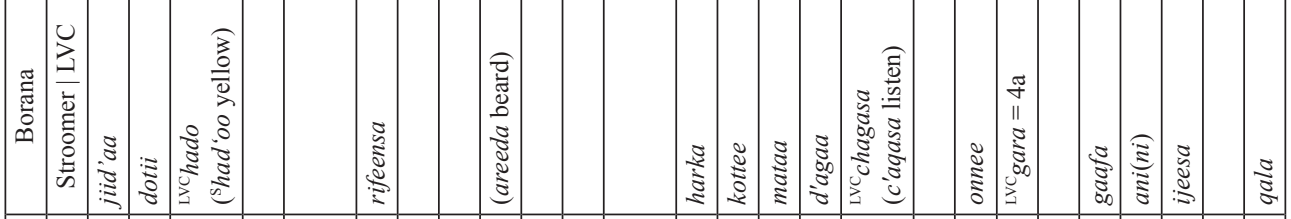

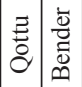

紊

登

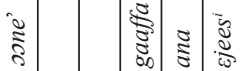

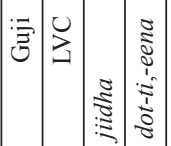

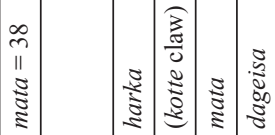

II

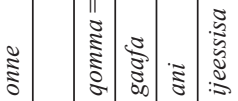

尊产

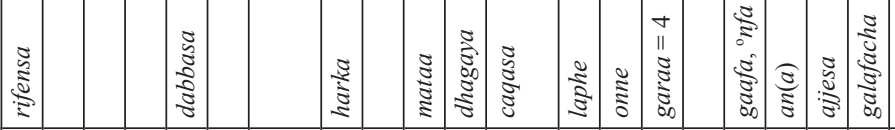

昰

竎

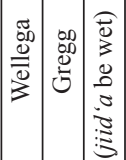

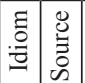

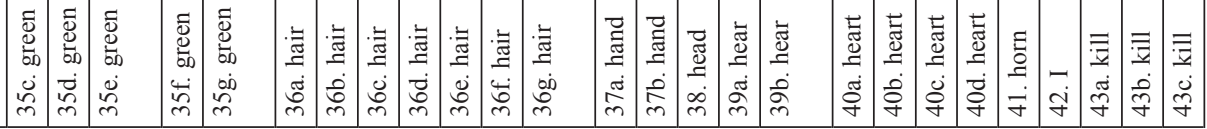




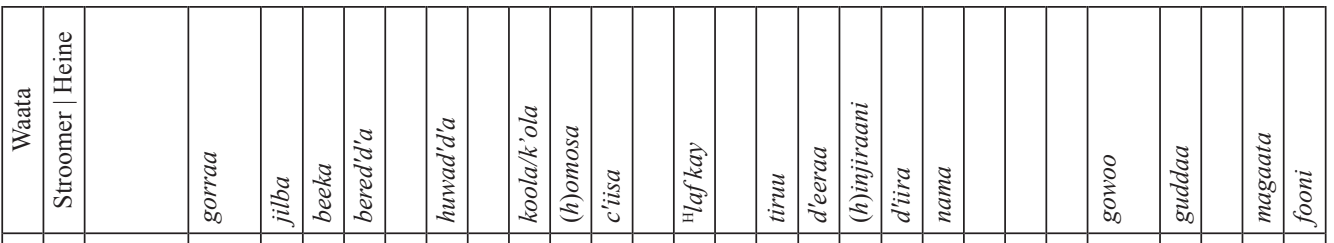

望鿷

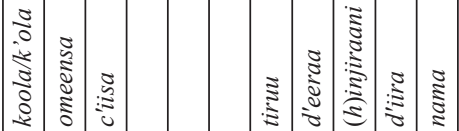

난

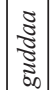

更

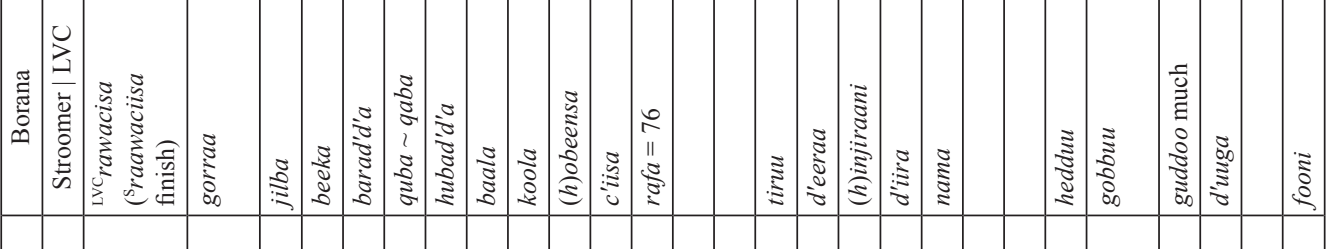

影腷

章

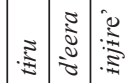

1

:

ร

言育

量

:

焉

$\sqrt[n]{2}$

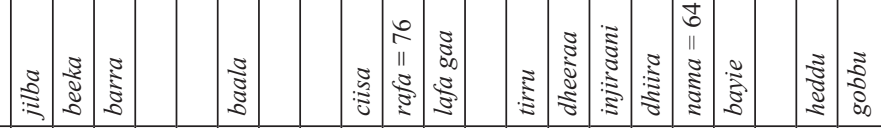

ฐ

莺 必

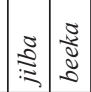

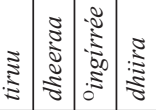

ฐँ

ฐ

善器

管

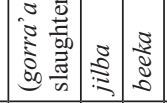

竎

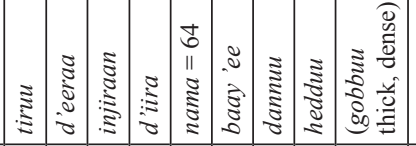

ฐ

佥影

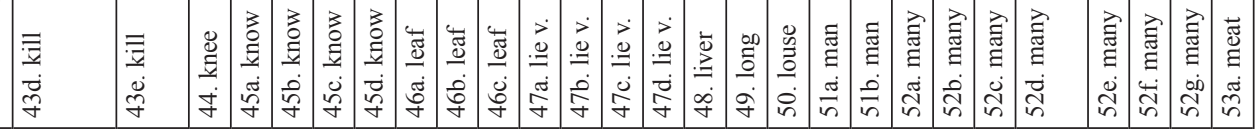




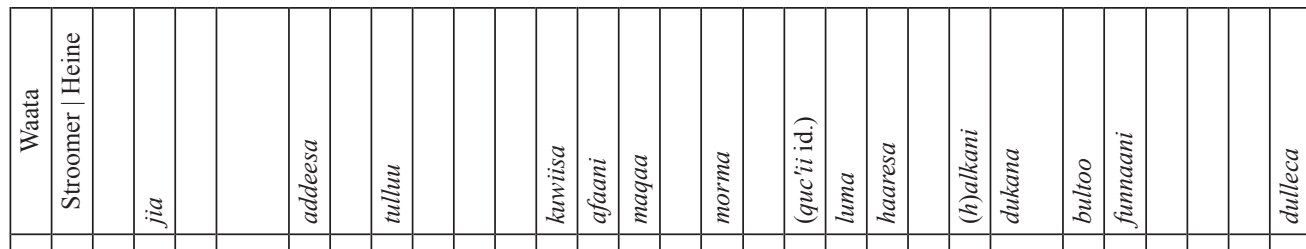

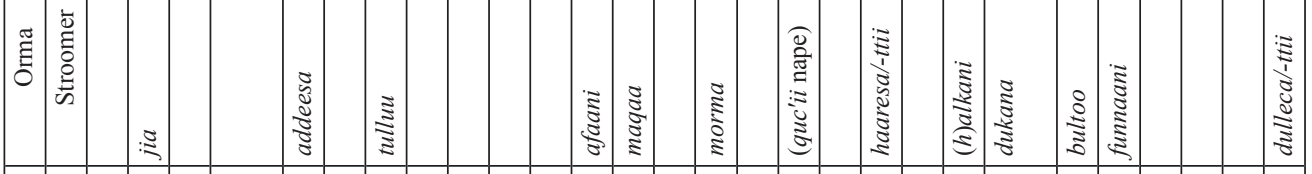

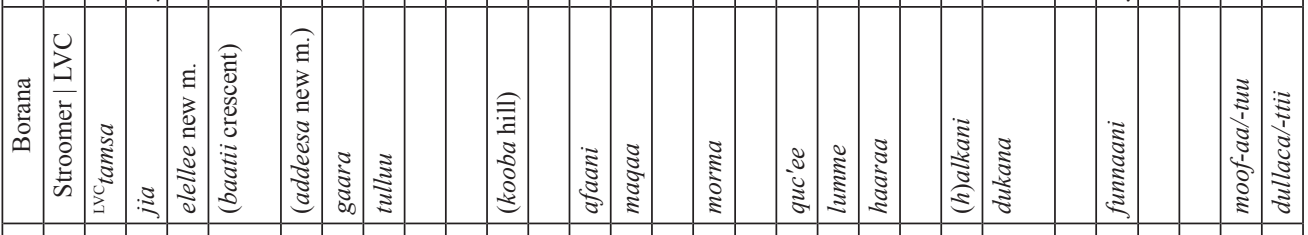

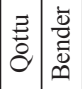

$\stackrel{0}{\vdots}$

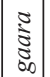

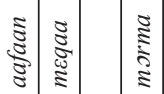

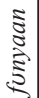

:

$:$

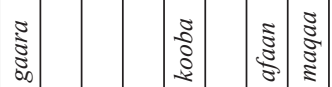

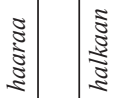

离

)

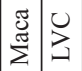

$: \cong$

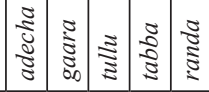

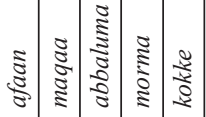

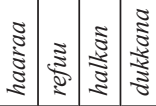

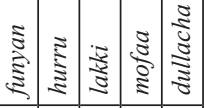

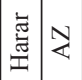

章

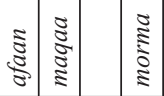

望

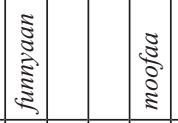

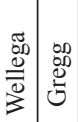

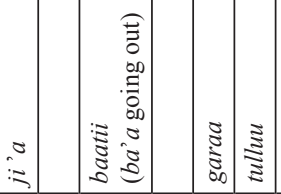

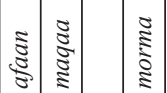

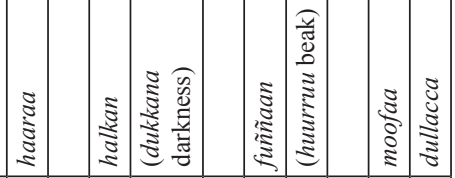

高敦

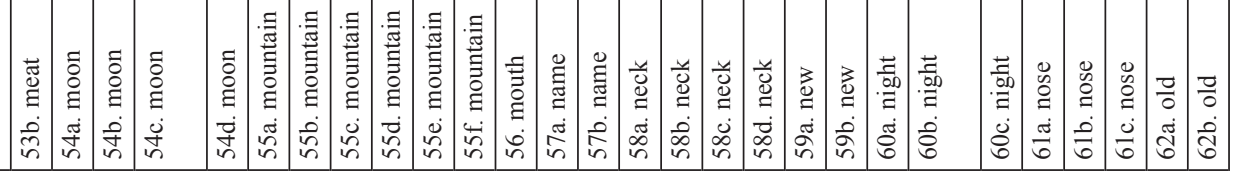




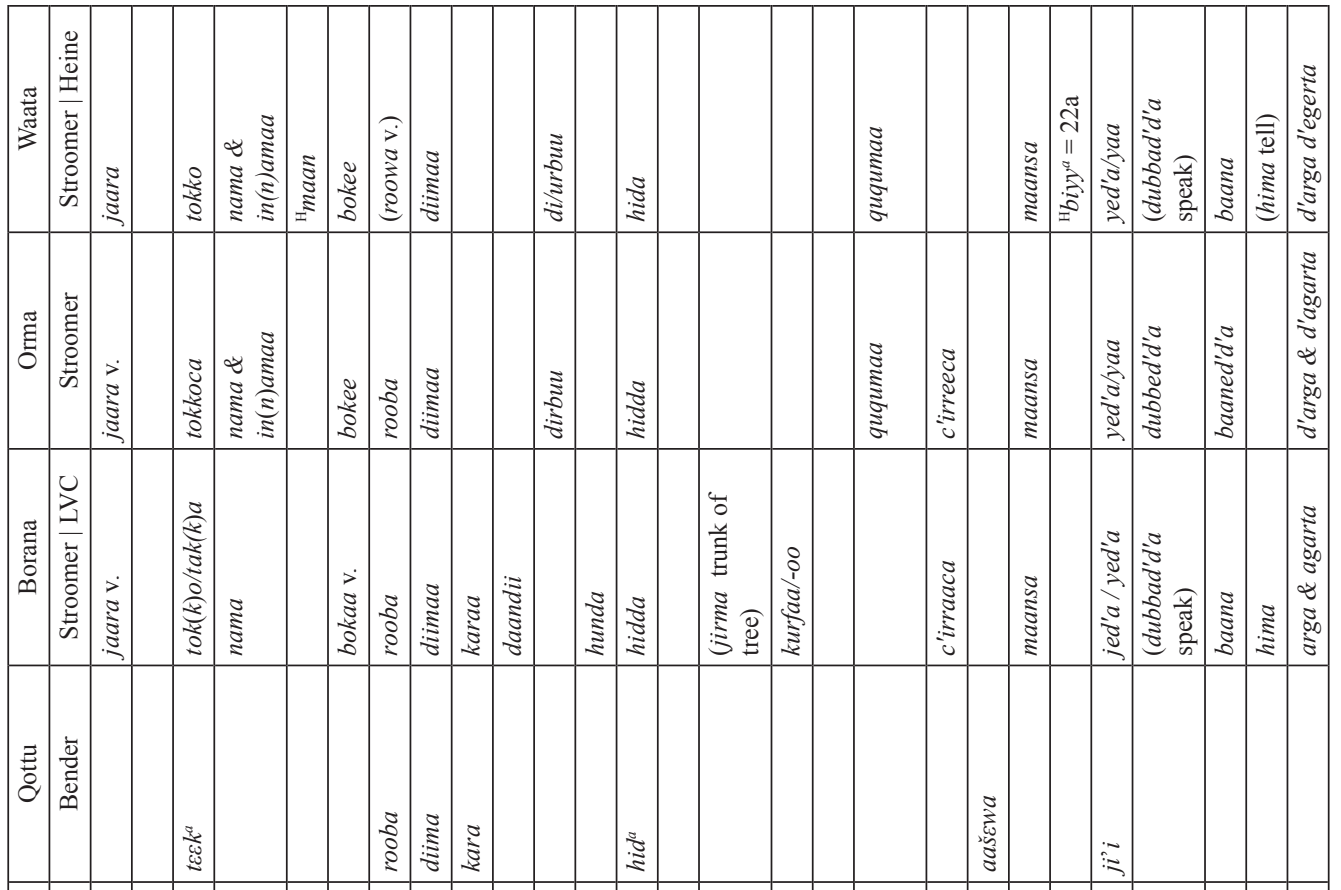

:

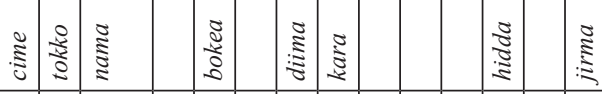

辛

咅

㩊微

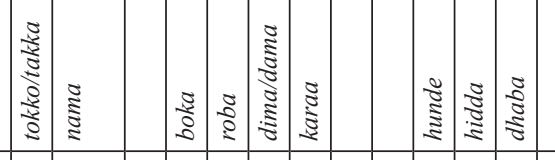

है

$\frac{\sqrt{2}}{2}$

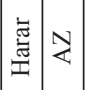
$\frac{\sqrt{2}}{2}$

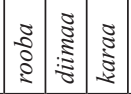
部离

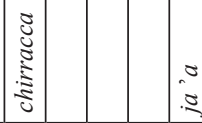

है

犃:

敛影

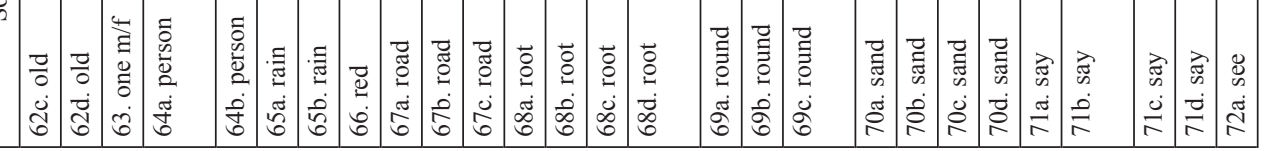




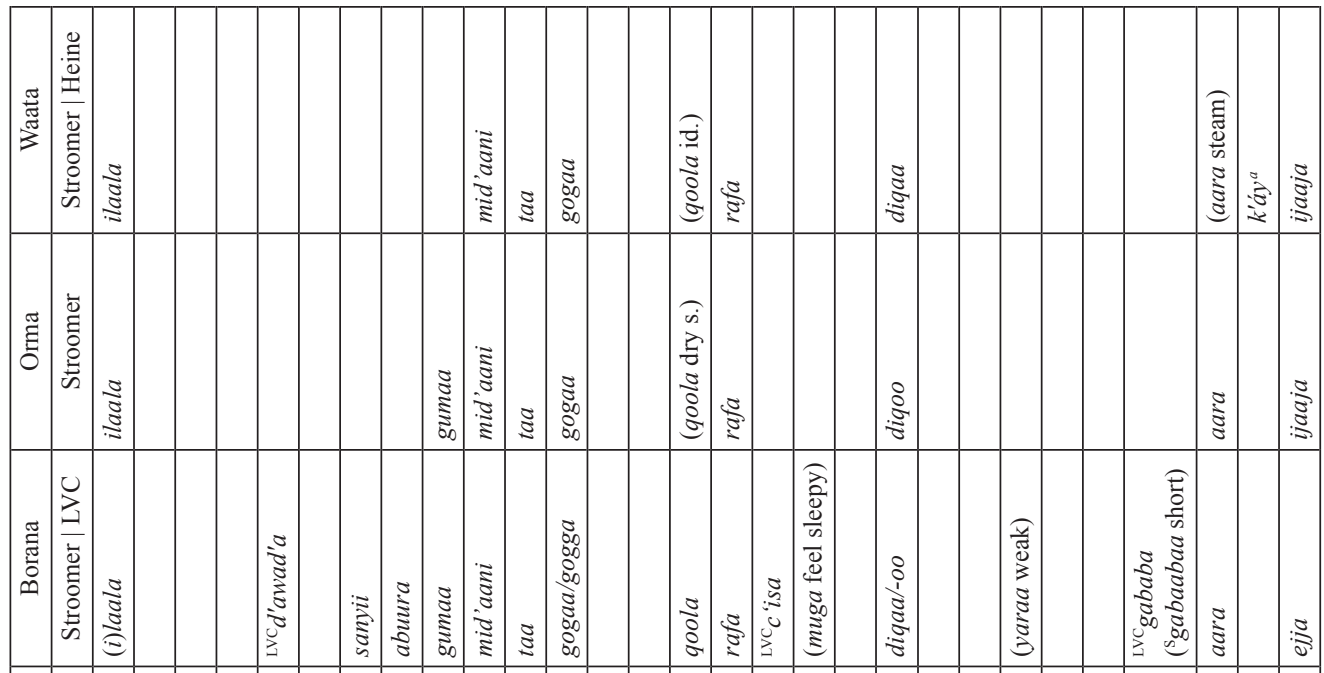

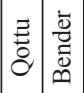
胥

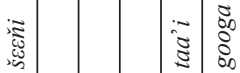

牙

$\frac{\pi}{2}$

:

:

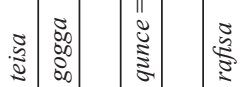

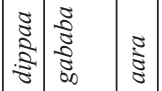

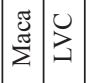

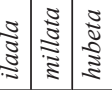

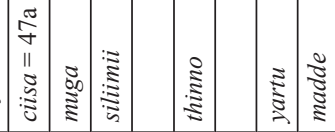

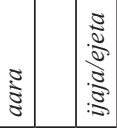

营过

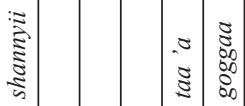

2

$\frac{3}{3}$

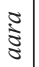

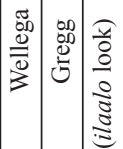

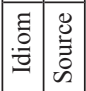

芯

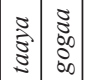

$\frac{3}{2}$

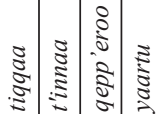

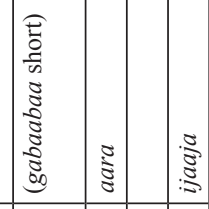

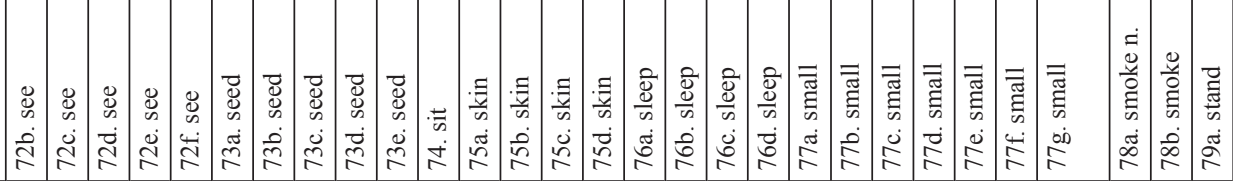




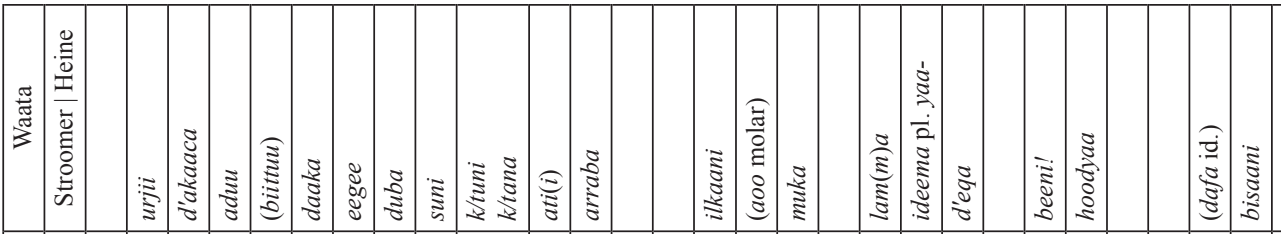

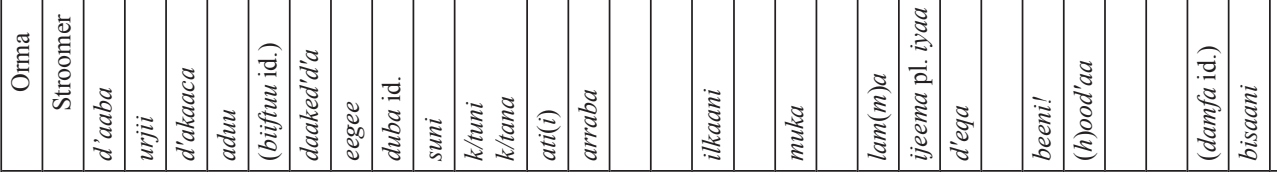

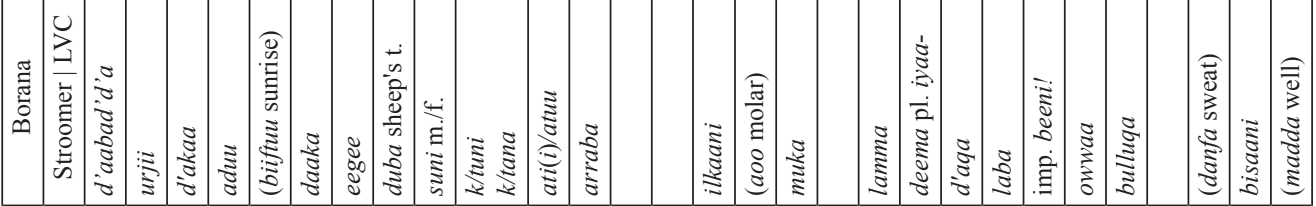

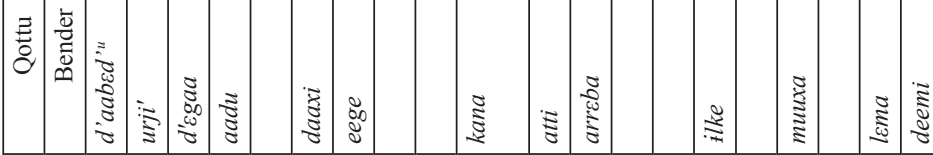

0
3
3
3
0

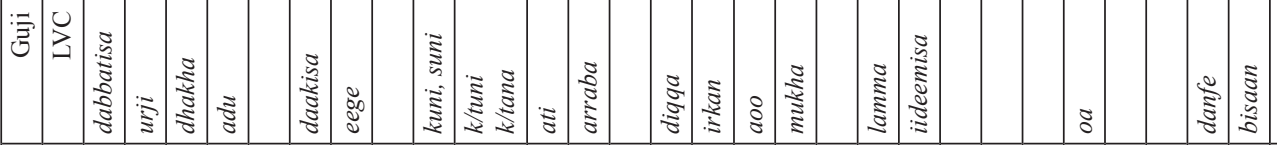

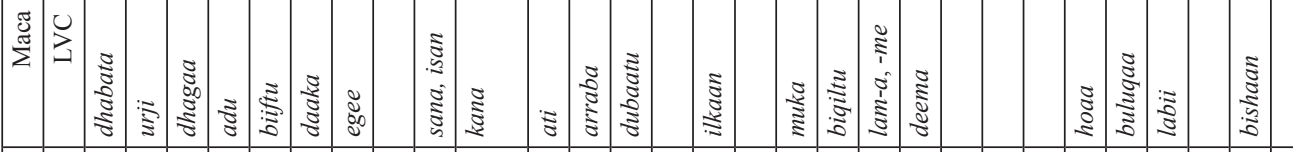

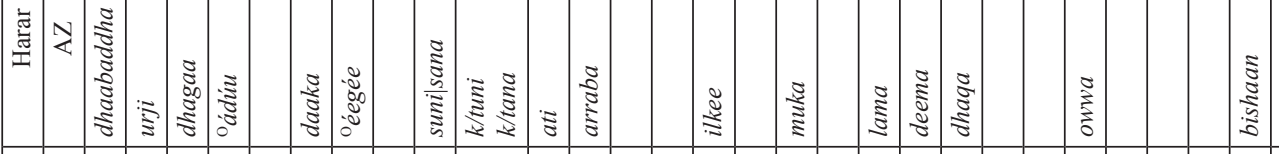

善䛠

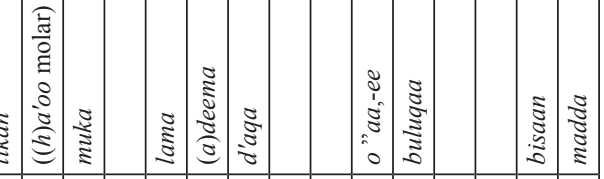

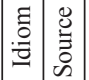

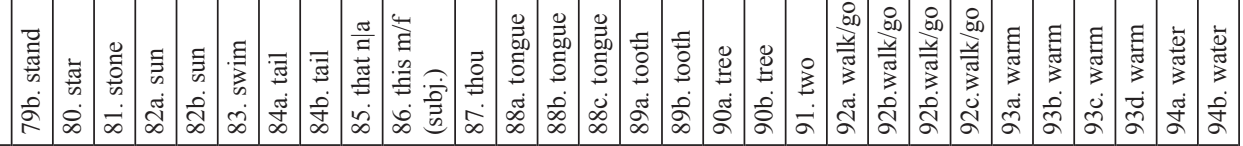




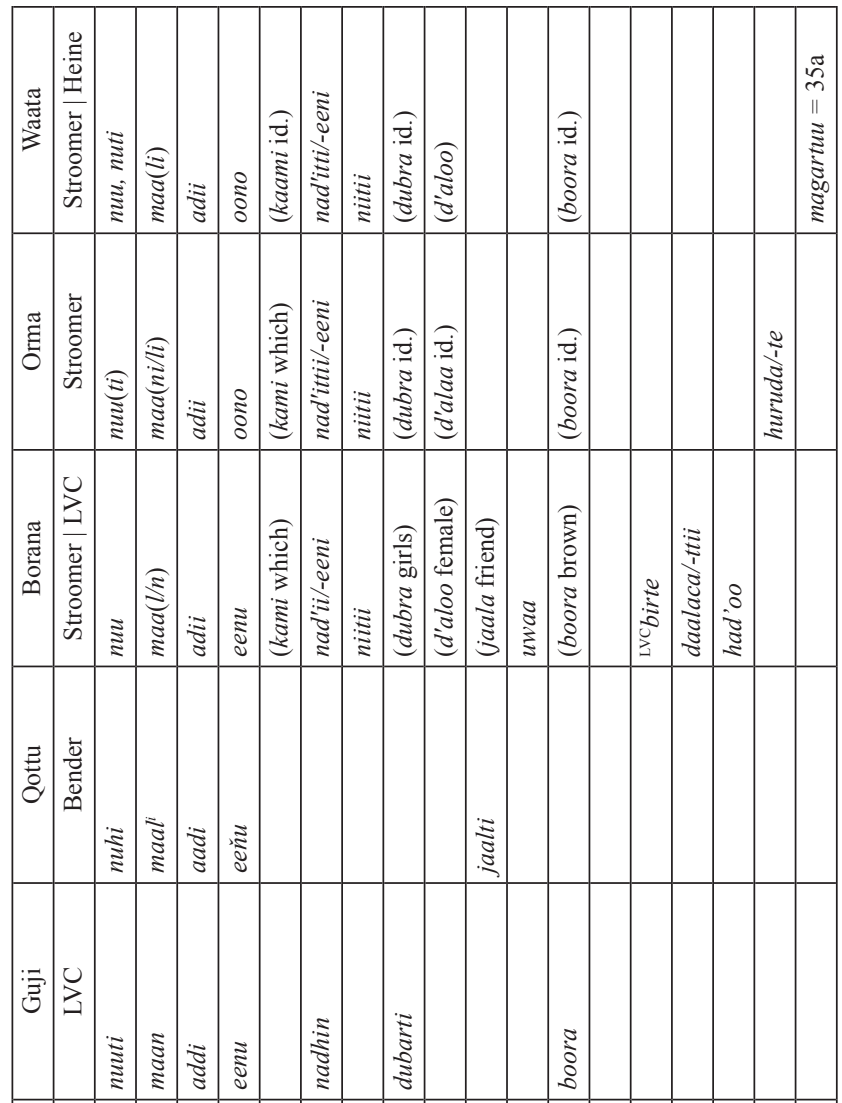

$\stackrel{\mathbb{E}}{\Sigma}$

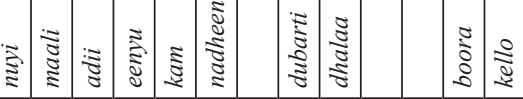

雚

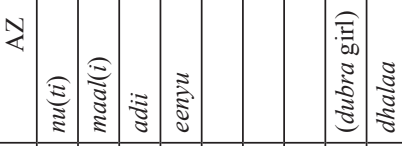

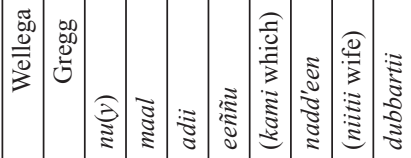

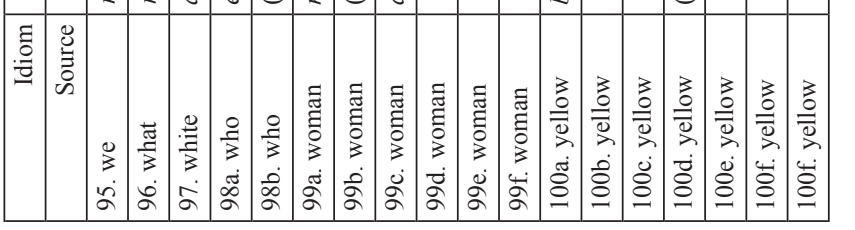

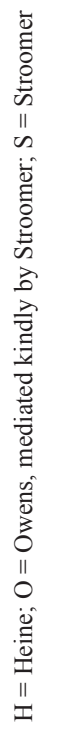




\section{ACKNOWLEDGMENT}

This study originated in cooperation with the Centre for the Interdisciplinary Research of Ancient Languages and Older Stages of Modern Languages (MSM 0021622435) at Masaryk University, Brno, and thanks to the grant No. IAA901640805.

\section{REFERENCES}

AZ = Ali Mohammed, ZABORSKI Andrzej. 1990. Handbook of the Oromo Language. Kraków: Ossolineum.

Bender M. Lionel. 1971. "Languages of Ethiopia. A New Lexicostatistic Classification and Some Problems of Diffusion." Anthropological Linguistics 13(5), 165-288.

Black Paul. 1974. Lowland East Cushitic: Subgrouping and Reconstruction. Yale University: PhD. Thesis.

BlažeK Václav. 1997. "Cushitic Lexicostatistics: The Second Attempt.” In: Afroasiatica Italiana. Studi Africanistici, Seria Etiopica 6. Napoli: Istituto Universitario Orientale, 171-188.

BlažeK Václav. 2006. East Cushitic Classification. Paper presented at the 36th "Colloquium on African languages and linguistics." Leiden: Ms.

BLAŽEK Václav, Záhořík Jan. 2008[09]. "Bayso: an Endangered Language of the Ethiopian Highlands." In: MACHALíK et al. 2008[09], 53-70.

Ehret Christopher; Ali M. Nuuh. 1984. "Soomaali Classification.” In: LaBaHn 1984: 201-269.

GragG Gene B. 1982. Oromo Dictionary. East Lansing: African Studies Center.

HeIne Bernd. 1976. "Notes on the Rendille Language (Kenya)." Afrika und Übersee 59, 176-223.

Heine Bernd. 1981. "The Waata Dialect of Oromo. Grammatical Sketch and Vocabulary.” In: Heine \& MöHLig 1981.

Heine Bernd, MöHlig Wilhelm J.G. (eds.). 1981. Language and Dialect Atlas of Kenya. Vol. 4. Berlin: Reimer.

Labahn Thomas (ed.). 1984. Proceedings of the Second International Congress of Somali Studies (Hamburg, august 1983). Vol. I: Linguistics and Literature. Hamburg: Buske.

LVC $=$ Leus Ton, VAN De Loo Joseph, CотTER George. 1992. A Vocabulary Oromo-English. Debre Zeït: Bole Press.

Machalík T., Mildnerová K., Záhořík J. (eds.). 2008[09]. Viva Africa. Proceedings of the 3rd International Conference on African Studies. Plzeň: Adéla.

Owens Jonathan. 1985. A Grammar of Harar Oromo (Northeastern Ethiopia). Hamburg: Buske.

Stroomer Harry. 1987. A Comparative Study of Three Southern Oromo Dialects in Kenya. Hamburg: Buske.

Stroomer Harry. 1995. A Grammar of Boraana Oromo (Kenya). Köln: Köppe.

Stroomer Harry. 2001. A Concise Vocabulary of Orma Oromo (Kenya). Orma - English / English - Orma. Köln: Köppe. 